\title{
Multidisciplinary Training Groups in a Psychiatric Hospital
}

\author{
Morris Nitsun (Principal Psychologist) Raymond Gledhill (Consultant Psychiatrist) and Rosalind Shanley (Senior \\ Psychologist), Goodmayes Hospital, Essex
}

Staff groups of various kinds are an important part of the life of a psychiatric hospital, yet there have been few attempts to document and assess their functioning. This would seem particularly important at present when the 'multidisciplinary approach' is regarded as an ideal in the treatment of the psychiatrically ill. Yet, many who have had experience of multidisciplinary groups are aware that they sometimes create as many problems as they solve. In this project, three multidisciplinary groups were formed from within the staff of a psychiatric hospital, cutting across the usual boundaries of ward and administrative teams, with the aim of studying the groups as representations of the institution and attempting to facilitate participants' functioning in the wider interdisciplinary setting.

\section{Formation of the groups}

The three group leaders, a consultant psychiatrist, the principal psychologist, and a senior psychologist, shared an interest in group processes and improving interdisciplinary relationships in the hospital. They decided to run the multidisciplinary training groups for a period of nine months, meeting once weekly for $1 \frac{1}{2}$ hours. Notices were sent to the various clinical departments, indicating that the project aimed at bringing together staff with the goal of achieving a greater understanding of their mutual problems as people and professionals. There were 55 replies, coming from all the departments circulated, although no consultant psychiatrist responded and administrative staff were not included (a mistake in hindsight).

A meeting was called of prospective participants and the leaders to discuss administrative aspects and interests. Participants completed questionnaires about their reason for joining the groups and their expectation. Recruitment of group members was without selection. The three groups were formed by the leaders drawing lots. Roughly equal representation of the different disciplines was achieved, but the groups were somewhat unbalanced for sex and seniority. The project ran from February to November 1975.

\section{Attendance}

There were a few drop-outs in the initial phase. Of the 44 who began, 12 were nurses, 10 social workers, 9 occupational therapists (mainly OT helpers), 5 doctors, 5 psychologists, 2 chaplains and one voluntary organizer. These figures correspond roughly to the ratio of staff in the hospital, except for nurses who are by far the largest group. Doctors were poorly represented, and those who participated were SHOs and one senior registrar. In contrast, occupational therapists, psychologists, chaplains, and to a lesser extent, social workers, were all represented. There was a fairly substantial drop-out rate. In spite of the requirement that participants should commit themselves for the full nine months, a number of people changed jobs in this period, there were a few trainees who rotated elsewhere and were unable to attend at the arranged time, while a small number decided not to continue. For the three groups combined $(n=44), 12$ participants dropped out at an early stage and 9 at about six months. Slightly more than half finished the groups. The two chaplains attended most regularly, followed by occupational therapists and psychologists. Doctors had a low initial drop-out rate, but several stopped about half-way. Social workers had the largest number of dropouts. They appeared to have the most realistic problems of attendance-most of them were based outside the hospital and had to come in for their groups, in addition to which their case-load often made urgent demands on their time.

\section{Progress of the groups}

The groups' functioning was similar to any small group, with phases of formation, consolidation and termination. In the initial phase there was considerable anxiety and even suspicion that this was a research study and that the project mattered more than the people. The research aspect was obvious, as participants completed questionnaires before and after the project as well as weekly. This met with strong resistance, both overt and covert. There was also uncertainty about the groups' direction, 'feeling out' of the leaders, and weighing up by participants of each other. Some participants felt strongly about having a particular leader. There was a lively, at times competitive, awareness between the groups, with participants frequently expressing curiosity about what other groups were doing and whether they were better than their own group.

An important early question was the type of group that would be most useful. Although the project referred to multidisciplinary training groups, the aim was not formal training but through group discussion and experience. The psychologists were interested in structured exercises and experimented with this in their first joint meeting, with useful results. Following this, however, they found in their separate groups that open-ended discussion served the groups' purpose better than structured interventions. This became the pattern of all three groups. Although the format was similar, differences in the leaders and in the groups composition produced marked differences in the groups' development.

The issues of work and the institution and related subjects such as authority, hierarchies and uniforms were of such central importance that they defined the characteristic development of each group. The psychiatrist's group dealt openly with these issues. One active member referred to two 
types of organization - the 'Sir' and the 'John'. The 'Sir' type was represented by the leader whom he identified with the upper echelons of the hierarchy; the 'John' organization represented himself and others who were placed lower down. These terms were adopted by other members. The group's dynamics revolved round these issues, and attendance was influenced by the resultant conflicts. Through examination of leadership roles, the psychiatrist found himself a group member as well as a leader and shared some of his own concerns. This contrasted with his role in patient groups which was more clearly one of leader.

The principal psychologist's group was different in that hospital politics were not openly discussed. There were two heads of department apart from himself (OT and nursing) which could have produced a political focus, particularly since there were long-standing difficulties between nursing and OT. However, the leader found it more appropriate to deal with here-and-now interactional issues, hoping that this itself might improve interdisciplinary relationships. This may have been less threatening to participants, as the group had a more stable membership than the other two groups. However, immediately following the project a spurt of interdisciplinary rivalry and criticism involving the psychology department suggested residual tensions that might have been dealt with more fully in the group.

The senior psychologist had a different 'authority' problem, as there was a narrower spread along the hierarchy in her group; no one was high up in the hierarchy, even though several members were designated 'senior'. The question was where the leadership lay, particularly since the group was ultimately mostly composed of women. There was some rivalry between the remaining male member and the senior psychologists for leadership. The group also examined the nurse-OT conflict, and the issue of group loyalities became a strong focus.

There were both similar conscious and underlying themes in the three groups. The issue of chaos vs. control, for example, was an important leitmotif. The principal psychologist felt that this underlined many other themes that developed in his group. Madness, for example, was seen as the feared chaos resulting from disorder, and authority and discipline as ways to prevent it. One participant referred to his group as the 'Baader-Meinhof group'. Although this was a casual remark, it may have expressed his fantasy of a group that could undermine the system. This sense of rebellion and fear of its consequences was common to all groups, and reflected the anxiety about assertiveness in the hospital, as well as in the groups.

The groups were not designed to be therapeutic, but participants did talk increasingly about themselves and seek solutions for personal difficulties. Such openness was difficult, because participants were colleagues, perhaps working closely together, and there was a fear of exposure. The expression of strong, irrational feelings and of curiosity about other members was probably more restrained than in a group of people who did not work together. Inhibition might also have been produced by differences in seniority, e.g. it seemed difficult for junior staff to reveal inadequacies and idiosyncrasies in the presence of senior colleagues, particularly if they were in the same department; the reverse also seemed to apply. Intradepartmental tensions were more difficult to discuss than interdepartmental ones, since the latter were more manifest in the hospital and participants could hide feelings about members of their own department by becoming involved in interdepartmental issues. Inhibited disclosure of personal problems also reflected the difficulty of dropping the therapist or 'helper' role and showing problems that might be the same as those of patients.

Similar defensive strategies were adopted in all three groups. There was a tendency to discusss absent members. Participants talked about practical solutions as opposed to psychological solutions, although it was suggested that these were not necessarily mutually exclusive and that psychological understanding might lead to more practical solutions. Another defence seemed to be to unconsciously engineer a set of rotating absences so that different people were present from week to week. While there were good reasons for absenteeism, this may also have been defensive. The result was that the groups became more like open groups, with changing membership, and discussion at times was fragmentary and superficial.

The termination phase brought an ambivalence about stopping, and a strong measure of anxiety and regret in some members. There was a feeling that the group had provided valuable support, some participants were concerned about not having anything in its place. The groups had succeeded in bringing together, in a novel way, the various disciplines. A number of these points are documented in the statement of one participant:

'The group gave me an opportunity to meet other members of the hospital staff and people outside the hospital in related work situations. To hear their views and difficulties, both in the job and personally, enabled me to see them as people, not just names or figures of authority. This also helped me to see myself more as a 'person' within the hospital setting. There are a lot of difficulties between different work areas which I hope these groups have helped to ease-but I would think that this would prevent people being fully at ease in talking about their feelings. To begin with I was very concerned not to let the OT down, a feeling which I lost fairly soon. I would like to meet and know more people and hope the groups will continue.'

\section{Quantitative study and discussion}

A study of participants' reactions was undertaken, using ratings of the group's value and specific criteria of its functioning. The small numbers and inconsistent completion by participants precluded full assessment of the data. The overall impression was that members found the groups valuable. They seemed to have high initial expectations and were disappointed during the project but more enthusiastic when judging it at the end. The areas in which gains were 
noted were: (1) awareness of, and contact with different professional roles; (2) knowledge and experience of group processes; and (3) self-awareness. A majority of participants felt that the groups should continue.

The project revealed an openness to questioning the status quo of relationships within the hospital, which was in contrast to the more general acceptance of institutional and hierarchical patterns of behaviour. It was felt to be a loss

\section{Reviews}

Behaviour Modification (1980): Report of a Joint Working Party to formulate Ethical Guidelines for the Conduct of Programmes of Behaviour Modification in the National Health Service. 1980. HMSO. £3.30.

This report, which could conveniently be termed the Zangwill Report, since the working party was chaired by Professor O. L. Zangwill, is described as a 'Consultative Document with Suggested Guidelines'. It is published jointly by the Royal College of Psychiatrists, the Royal College of Nursing and the British Psychological Society, and is based on the work of a multidisciplinary group, 16 strong, who conducted a nationwide survey, visited seven hospitals and received written or oral evidence from a comprehensive list of organizations and individuals involved in the field. The working party was originally set up at the request of Sir Keith Joseph, when he was Secretary of State for Social Services and has a short, polite foreword by Patrick Jenkin. With this prestigious background it requires careful explanation to account for the fact that on the whole this is a disappointing piece of work.

The most obvious reason is that it is out of date. The working party first met on 12 March 1974, started their survey two months later, and made their visits in April 1975. It is not clear why it has taken over five years since then for their findings to be published; this excessive delay is particularly unfortunate in the field of behaviour modification. Recent developments and changes in approach make much of the working party's discussion of historical interest only. Thus, considerable attention is paid to traditional aversion therapy which is nowadays scarcely ever used. When it is, the aversive stimuli are invariably self-administered, thus completely under the patient's control. From the ethical point of view, this poses few, if any, of the problems of the traditional treatment which was modelled on the work done on Pavlov's unfortunate dogs. Likewise, much of the report is concerned with token economies and in particular the ethical implications of controlling rewards, such as money, cigarettes, and freedom of movement which may be considered a basic part of any patient's rights. Again, the enthusiasm for strictly-run token economies has waned, that no consultant psychiatrist (other than the one leader) participated, that nursing staff were not well represented, and that administrative staff were not included. Although the study had no pretensions of changing the functioning of the hospital, we believe that in some respects staff relationships improved (e.g. the long-standing nursing-OT controversy). Whether this bears any causal link with the project is not clear.

largely because of lack of experimental evidence to support their efficacy. Social reinforcement, high levels of staffpatient interaction and ignoring unwanted behaviour are likely to be the ingredients of an up-to-date operant programme. In 1981 political and financial difficulties dwarf any ethical dilemmas involved in running such systems. There is little point in agonizing about the ethics of a treatment programme, if you cannot afford to employ the staff to run the ward at more than a level of basic containment. The report admits that in many parts of the country 'basic rights barely reach acceptable standards'. Surely this, rather than the misplaced enthusiasm of a handful of poorly trained staff, is the real scandal that we need to tackle.

The second point to be aware of is that the working party chose to concentrate on behaviour modification applied to groups as its principal concern. In other words, their arguments largely concern operant programmes for chronic psychosis and the mentally handicapped. Most behavioural psychotherapists spend the bulk of their time in the outpatient treatment of neuroses, a style of work which involves different ethical issues. Since many non-specialists are unaware of the wide range of different 'behavioural' treatments, there is a danger that attempts may be made to extrapolate the conclusions of this report, which concerns a narrow, idiosyncratic form of treatment to a much wider field - the equivalent of extending the findings of the Committee on Review of Medicines concerning minor tranqullizers to the whole range of psycho-active drugs.

Next, although mentioned in passing, the working party fails to show why the ethical issues posed by behaviour modification are in need of more urgent, special, study than similar issues in any other areas of psychotherapy or psychiatry. What about the ethical implications of ward groups run by staff with little training and even less supervision, in which mysterious 'interpretations' or hurtful confrontations are imposed upon the patients without the consent of either the patients or their relatives? Similarly, if, as the report quite reasonably recommends, it is desirable that behaviour modification should be explicit and open to public scrutiny, is it not equally important to apply the same criteria to psychoanalysis? 